\title{
Dissipative effects on the mean square displacement of an oscillator
}

\author{
X.L. $\mathrm{Li}^{\mathrm{a}}$, G.W. Ford ${ }^{\mathrm{b}}$ and R.F. O'Connell ${ }^{\mathrm{a}}$ \\ ${ }^{2}$ Department of Physics and Astronomy, Louisiana State University, \\ Baton Rouge, LA 70803-4001, USA \\ ${ }^{b}$ Department of Physics, University of Michigan, Ann Arbor, MI 48109-1120, USA
}

Received 4 June 1992

The localization of an oscillator in a dissipative environment may be characterized by its mean square displacement. In the case of a general heat bath, we write down an expression for this quantity as an integral involving the imaginary part of the generalized susceptibility. This integral is evaluated explicitly in the case of both Ohmic and blackbody radiation heat baths, leading us to the conclusion that localization is enhanced by an increase of dissipation or oscillator frequency (binding) and by a decrease in temperature.

\section{Introduction}

Dissipative effects are ubiquitous in many areas of physics. In some previous publications [1,2] we argued the merits of treating an exactly solvable model of a heat bath, which we referred to as the IO model [2]. In particular, this model can be shown to describe many kinds of dissipative environments, such as Ohmic heat baths or the physically important case of a blackbody radiation heat bath [2].

In order to gain further insight into the nature of the IO model, we are motivated to examine in detail the effect of dissipation on the mean square displacement or, equivalently, the equal-time position autocorrelation function. In particular, such a quantity may be used to calculate the effect of dissipation on the localization of an oscillator. Some investigations have already been carried out for the case of an Ohmic heat bath [3]. Our purpose here is to expand these investigations but, more important, to extend these considerations to the case of a blackbody radiation heat bath.

A powerful tool for solving the problem of the interaction of a quantum system with a heat bath is the generalized quantum Langevin equation, which, 
for a particle of mass $m$ in a harmonic potential well with spring constant $K$, takes the form $[1,2]$

$$
m \ddot{x}+\int_{-\infty}^{t} \mathrm{~d} t^{\prime} \mu\left(t-t^{\prime}\right) \dot{x}\left(t^{\prime}\right)+K x=F(t) .
$$

This is an equation for the time-dependent Heisenberg operator $x(t)$. The coupling with the heat bath corresponds to two terms: an operator-valued random force $F(t)$ with mean zero, and a mean force characterized by a memory function $\mu(t)$. Forming the Fourier transform of (1.1), we obtain

$$
\tilde{x}(\omega)=\alpha(\omega) \tilde{F}(\omega),
$$

where the superposed tilde denotes the Fourier transform, and $\alpha(\omega)$ is the generalized susceptibility (a c-number) given by

$$
\alpha(\omega)=\left[-m \omega^{2}+K-i \omega \tilde{\mu}(\omega)\right]^{-1},
$$

where

$$
\tilde{\mu}(\omega)=\int_{0}^{\infty} \mathrm{d} t \mu(t) \mathrm{e}^{\mathrm{i} \omega t}, \quad \operatorname{Im} \omega>0,
$$

called the spectral distribution, is the Fourier transform of the memory function. It is analytic in the upper half of the $\omega$ plane and its real part is positive on the real axis [2]. Such functions are termed positive functions. It can be shown that $-i \omega \alpha(\omega)$ is also a positive function provided that $m$ and $K$ are positive $[1,2]$. It follows that

$$
\operatorname{Im} \alpha(\omega)>0 \quad \text { for } \omega>0 .
$$

The generalized susceptibility plays an important role in determining the dynamics of the system. On applying the fluctuation-dissipation theorem, we immediately obtain $[3,4]$

$$
\left\langle x^{2}\right\rangle=\frac{\hbar}{\pi} \int_{0}^{\infty} \mathrm{d} \omega \operatorname{coth}\left(\frac{\hbar \omega}{2 k T}\right) \operatorname{Im}\left[\alpha\left(\omega+\mathrm{i} 0^{\prime}\right)\right] .
$$

Since the factor $\operatorname{coth}(\hbar \omega / 2 k T)$ in the integrand of (1.6) is a monotonically increasing function of $T$, it follows, by (1.5), that $\left\langle x^{2}\right\rangle$ is also a monotonically increasing function of $T$, i.e., 


$$
\frac{\partial}{\partial T}\left\langle x^{2}\right\rangle>0
$$

In other words, as we might expect, in the case of an arbitrary spectral distribution, higher temperatures favor delocalization.

In sections 2 and 3, we shall calculate in detail, using (1.6), the mean square oscillator displacement and its derivatives for both Ohmic and blackbody radiation heat baths, at zero and non-zero temperatures. In section 4 , we present our conclusions.

\section{Ohmic heat bath}

This is the simplest type of heat bath with $\tilde{\mu}(\omega)=m \gamma$, a constant independent of the frequency $\omega$. The corresponding generalized susceptibility, by (1.3) is

$$
\alpha(\omega)=\frac{1}{m\left(\omega_{0}^{2}-\omega^{2}-\mathrm{i} \gamma \omega\right)},
$$

where

$$
\omega_{0}^{2}=\frac{K}{m} .
$$

Then

$$
\begin{aligned}
\operatorname{Im} \alpha(\omega) & =\frac{\gamma \omega}{m\left[\left(\omega^{2}-\omega_{0}^{2}\right)^{2}+\gamma^{2} \omega^{2}\right]} \\
& =\frac{\omega}{2 m} \operatorname{Im}\left[\frac{1}{\sqrt{\omega_{0}^{2}-\frac{1}{4} \gamma^{2}}}\left(\frac{1}{\omega^{2}+\omega_{2}^{2}}-\frac{1}{\omega^{2}+\omega_{1}^{2}}\right)\right],
\end{aligned}
$$

where $\omega_{1,2}=\frac{1}{2} \gamma \pm \mathrm{i} \sqrt{\omega_{0}^{2}-\frac{1}{4} \gamma^{2}}$.

At zero temperature $(T=0), \operatorname{coth}(\hbar \omega / 2 k T)=1$. Inserting this and $(2.3)$ in (1.6), we obtain the mean square oscillator displacement at zero temperature [3],

$$
\begin{aligned}
\left.\left\langle x^{2}\right\rangle\right|_{T=0} & =\frac{\hbar}{\pi} \int_{0}^{\infty} \mathrm{d} \omega \operatorname{Im}[\alpha(\omega)]=\frac{\hbar}{2 \pi m} \operatorname{Im}\left[\frac{1}{\sqrt{\omega_{0}^{2}-\frac{1}{4} \gamma^{2}}} \ln \left(\frac{\omega_{1}}{\omega_{2}}\right)\right] \\
& =\frac{\hbar}{\pi m \sqrt{\omega_{0}^{2}-\frac{1}{4} \gamma^{2}}} \tan ^{-1}\left(\frac{2}{\gamma} \sqrt{\omega_{0}^{2}-\frac{1}{4} \gamma^{2}}\right) \quad \text { if } \omega_{0}>\frac{1}{2} \gamma \\
& =\frac{\hbar}{\pi m \sqrt{\omega_{0}^{2}-\frac{1}{4} \gamma^{2}}} \sin ^{-1}\left(\sqrt{\omega_{0}^{2}-\frac{1}{4} \gamma^{2}} / \omega_{0}\right)
\end{aligned}
$$


and

$$
=\frac{\hbar}{2 \pi m \sqrt{\frac{1}{4} \gamma^{2}-\omega_{0}^{2}}} \ln \left(\frac{\frac{1}{2} \gamma+\sqrt{\frac{1}{4} \gamma^{2}-\omega_{0}^{2}}}{\frac{1}{2} \gamma-\sqrt{\frac{1}{4} \gamma^{2}-\omega_{0}^{2}}}\right) \quad \text { if } \quad \omega_{0}<\frac{1}{2} \gamma .
$$

From (2.4), we see that for $\omega_{0} \ll \frac{1}{2} \gamma$ this function reduces to $(2 \hbar / \pi m \gamma) \ln (\gamma /$ $\left.\omega_{0}\right)$, for $\frac{1}{2} \gamma \ll \omega_{0}$ it reduces to $\hbar / 2 m \omega_{0}$ and for $\frac{1}{2} \gamma=\omega_{0}$ it equals $\hbar / \pi m \omega_{0}=2 \hbar$ / $\pi m \gamma$. These results already appear in the work of ref. [3] (p. 437). Our purpose here is to use them to investigate the detailed behavior of the mean square displacement on the parameters $\gamma$ and $K$ and eventually compare them with the corresponding results in the case of a blackbody radiation heat bath.

It is then straightforward to check that

$$
\begin{aligned}
& \frac{\partial}{\partial \gamma}\left\langle x^{2}\right\rangle= \\
& \begin{cases}\frac{\hbar \gamma}{4 \pi m\left(\omega_{0}^{2}-\frac{1}{4} \gamma^{2}\right)^{3 / 2}}\left[\tan 1\left(\frac{2}{\gamma} \sqrt{\omega_{0}^{2}-\frac{1}{4} \gamma^{2}}\right)-\frac{2}{\gamma} \sqrt{\omega_{0}^{2}-\frac{1}{4} \gamma^{2}}\right] & \text { if } \omega_{0}>\frac{1}{2} \gamma, \\
\frac{\hbar \gamma}{4 \pi m\left(\frac{1}{4} \gamma^{2}-\omega_{0}^{2}\right)^{3 / 2}}\left[\frac{2}{\gamma} \sqrt{\frac{1}{4} \gamma^{2}-\omega_{0}^{2}}-\frac{1}{2} \ln \left(\frac{\left.\left.\frac{1}{2} \gamma+\sqrt{\frac{1}{4} \gamma^{2}-\omega_{0}^{2}}\right)\right]}{\frac{1}{2} \gamma-\sqrt{\frac{1}{4} \gamma^{2}-\omega_{0}^{2}}}\right]\right. & \text { if } \omega_{0}<\frac{1}{2} \gamma,\end{cases}
\end{aligned}
$$

and that

$$
\begin{aligned}
& \frac{\partial}{\partial K}\left\langle x^{2}\right\rangle= \\
& \begin{cases}\frac{\hbar}{2 \pi m^{2}\left(\omega_{0}^{2}-\frac{1}{4} \gamma^{2}\right)^{3 / 2}}\left[\frac{\gamma}{2 \omega_{0}^{2}} \sqrt{\omega_{0}^{2}-\frac{1}{4} \gamma^{2}}-\tan ^{-1}\left(\frac{2}{\gamma} \sqrt{\omega_{0}^{2}-\frac{1}{4} \gamma^{2}}\right)\right] & \text { if } \omega_{0}>\frac{1}{2} \gamma, \\
\frac{\hbar}{2 \pi m^{2}\left(\frac{1}{4} \gamma^{2}-\omega_{0}^{2}\right)^{3 / 2}}\left[\frac{1}{2} \ln \left(\frac{\frac{1}{2} \gamma+\sqrt{\frac{1}{4} \gamma^{2}-\omega_{0}^{2}}}{\frac{1}{2} \gamma-\sqrt{\frac{1}{4} \gamma^{2}-\omega_{0}^{2}}}\right)-\frac{\gamma}{2 \omega_{0}^{2}} \sqrt{\frac{1}{4} \gamma^{2}-\omega_{0}^{2}}\right] & \text { if } \omega_{0}<\frac{1}{2} \gamma .\end{cases}
\end{aligned}
$$

Both derivatives may be shown to be negative by use of the inequalities: $\tan ^{-1} x<x \quad(x>0) \quad$ and $\frac{1}{2} \ln [(1+x) /(1-x)]>x \quad(0<x<1)$ in $(2.5)$; $\tan ^{-1} x>x /\left(1+x^{2}\right)(x>0)$ and $\frac{1}{2} \ln [(1+x) /(1-x)]<x /\left(1-x^{2}\right)(0<x<1)$ in (2.6), respectively. Thus, we conclude that, at zero temperature, localization becomes enhanced due to increasing of $\gamma$ (i.e. damping) or $K$ (i.e. binding).

Next we consider the case of non-zero temperature. Since

$$
\operatorname{coth}\left(\frac{\hbar \omega}{2 k T}\right)=1+\frac{2}{\exp (\hbar \omega / k T)-1},
$$


denoting the temperature dependent part of $\left\langle x^{2}\right\rangle$ as $\Delta\left\langle x^{2}\right\rangle$, we have

$$
\begin{aligned}
\Delta\left\langle x^{2}\right\rangle & \equiv\left\langle x^{2}\right\rangle-\left.\left\langle x^{2}\right\rangle\right|_{T=0} \\
& =\frac{2 \hbar}{\pi} \int_{0}^{\infty} \mathrm{d} \omega \frac{1}{\exp (\hbar \omega / k T)-1} \operatorname{Im}[\alpha(\omega)] .
\end{aligned}
$$

Using (2.3), this becomes

$$
\Delta\left\langle x^{2}\right\rangle=\frac{\hbar}{2 \pi m} \operatorname{Im}\left\{\frac{1}{\sqrt{\omega_{0}^{2}-\frac{1}{4} \gamma^{2}}}\left[\ln \left(\frac{z_{2}}{z_{1}}\right)+\psi\left(z_{1}\right)-\psi\left(z_{2}\right)+\frac{1}{2 z_{1}}-\frac{1}{2 z_{2}}\right]\right\},
$$

where

$$
z_{1,2} \equiv \frac{\hbar}{2 \pi k T} \omega_{1,2}
$$

and we have used the formula

$$
2 \int_{0}^{\infty} \frac{t \mathrm{~d} t}{[\exp (2 \pi t)-1]\left(t^{2}+z^{2}\right)}=\ln z-\psi(z)-\frac{1}{2 z} \quad\left(|\arg z|<\frac{1}{2} \pi\right),
$$

where $\psi(z)=\mathrm{d} \ln \Gamma(z) / \mathrm{d} z$ is the logarithmic derivative of the gamma function [5].

Hence

$$
\begin{aligned}
\left\langle x^{2}\right\rangle & =\left.\left\langle x^{2}\right\rangle\right|_{T=0}+\Delta\left\langle x^{2}\right\rangle \\
& =\frac{\hbar}{2 \pi m} \operatorname{Im}\left[\frac{1}{\sqrt{\omega_{0}^{2}-\frac{1}{4} \gamma^{2}}}\left(\psi\left(z_{1}\right)-\psi\left(z_{2}\right)+\frac{1}{2 z_{1}}-\frac{1}{2 z_{2}}\right)\right] .
\end{aligned}
$$

In the high-temperature limit, $z_{1,2} \ll 1$, this expression becomes

$$
\left\langle x^{2}\right\rangle=\frac{k T}{m \omega_{0}^{2}}+\frac{\hbar^{2}}{12 m k T}-\frac{\hbar^{3} \gamma \zeta(3)}{4 \pi^{3} m(k T)^{2}}+\cdots,
$$

where $\zeta(3)=1.202 \ldots(\zeta(n)$ is the Riemann zeta function). The leading term in (2.13) is the familiar classical result.

In the low-temperature limit, $z_{1.2} \gg 1,(2.12)$ becomes

$$
\left\langle x^{2}\right\rangle=\frac{\hbar}{\pi m \sqrt{\omega_{0}^{2}-\frac{1}{4} \gamma^{2}}} \tan ^{-1}\left(\frac{2}{\gamma} \sqrt{\omega_{0}^{2}-\frac{1}{4} \gamma^{2}}\right)+\frac{\pi \gamma(k T)^{2}}{3 \hbar m \omega_{0}^{4}}+\cdots .
$$


Taking the derivatives of (2.12), one can readily show that

$$
\begin{aligned}
\frac{\partial}{\partial \gamma}\left\langle x^{2}\right\rangle= & -\frac{\hbar}{4 \pi m\left(\omega_{0}^{2}-\frac{1}{4} \gamma^{2}\right)} \\
& \times \operatorname{Re}\left(\frac{z_{2}+z_{1}}{z_{2}-z_{1}}\left[\psi\left(z_{1}\right)-\psi\left(z_{2}\right)\right]+z_{1} \psi^{\prime}\left(z_{1}\right)+z_{2} \psi^{\prime}\left(z_{2}\right)\right),
\end{aligned}
$$

and that

$$
\begin{aligned}
\frac{\partial}{\partial K}\left\langle x^{2}\right\rangle= & -\frac{\hbar^{2}}{4 \pi^{2} m^{2} k T\left(\omega_{0}^{2}-\frac{1}{4} \gamma^{2}\right)} \\
& \times \operatorname{Re}\left[\frac{\psi\left(z_{1}\right)-\psi\left(z_{2}\right)}{z_{1}-z_{2}}-\frac{1}{2}\left[\psi^{\prime}\left(z_{1}\right)+\psi^{\prime}\left(z_{2}\right)\right]+\frac{1}{4}\left(\frac{1}{z_{1}}-\frac{1}{z_{2}}\right)^{2}\right] .
\end{aligned}
$$

By means of the partial fractional expansion of $\psi(z)$,

$$
\psi(z)=-\delta-\frac{1}{z}+\sum_{n=1}^{\infty} \frac{z}{n(n+z)} \quad(z \neq 0,-1,-2,-3, \ldots)
$$

where $\delta=0.57721 \ldots$ is the Euler constant (we have used an unconventional symbol here to avoid the confusion with the friction constant $\gamma$ ), (2.15) may be written

$$
\frac{\partial}{\partial \gamma}\left\langle x^{2}\right\rangle=-\frac{\hbar^{3}}{4 \pi^{3} m(k T)^{2}} \operatorname{Re}\left(\sum_{n=1}^{\infty} \frac{n}{\left(n+z_{1}\right)^{2}\left(n+z_{2}\right)^{2}}\right) .
$$

Similarly

$$
\begin{aligned}
\frac{\partial}{\partial K}\left\langle x^{2}\right\rangle= & -\frac{\hbar^{4}}{8 \pi^{4} m^{2}(k T)^{3}} \\
& \times \operatorname{Re}\left(\frac{1}{2\left(z_{1} z_{2}\right)^{2}}+\sum_{n=1}^{\infty} \frac{1}{\left(n+z_{1}\right)^{2}\left(n+z_{2}\right)^{2}}\right) .
\end{aligned}
$$

Since from (2.3) and (2.10), $z_{1}$ and $z_{2}$ are either complex conjugates of each other (if $\omega_{0}>\frac{1}{2} \gamma$ ) or two real positive quantities (if $\omega_{0}<\frac{1}{2} \gamma$ ), the summands within the large parentheses in (2.18) and (2.19) are all real positive quantities. Therefore we conclude that

$$
\frac{\partial}{\partial \gamma}\left\langle x^{2}\right\rangle<0
$$

and that 


$$
\frac{\partial}{\partial K}\left\langle x^{2}\right\rangle<0
$$

We conclude that in the case of an Ohmic heat bath, at arbitrary temperature, the mean square displacement of a quantum oscillator monotonically decreases (so that the oscillator becomes more localized) with increasing $\gamma$ or $K$.

It is also of interest to check the $\gamma \rightarrow 0^{+}$limit of $\left\langle x^{2}\right\rangle$. In the absence of a heat bath, $(2.10)$ becomes $z_{1,2}= \pm \mathrm{i} \hbar \omega_{0} / 2 \pi k T$. By using the recursion formula, $\psi(z+1)=\psi(z)+1 / z$, and the reflection formula, $\psi(1-z)=\psi(z)+\pi \cot (\pi z)$, (2.12) can be reduced to

$$
\left\langle x^{2}\right\rangle=\frac{\hbar}{2 m \omega_{0}} \operatorname{coth}\left(\frac{\hbar \omega_{0}}{2 k T}\right),
$$

which is exactly the result for a free quantum oscillator at temperature $T$.

\section{Blackbody radiation heat bath}

In this case, the spectral distribution function is $[1,2]$

$$
\tilde{\mu}(\omega)=2 e^{2} \Omega^{2} \omega / 3 c^{3}(\omega+\mathrm{i} \Omega),
$$

where $\Omega$ is a large cutoff frequency.

Here one must be careful to go to the large-cutoff limit only after the completion of the integral in (1.6). The order can be of importance in some cases, as will be shown later in the calculation. (In particular, note the $\ln m$ in (3.13) which is divergent in the large cutoff limit).

Putting (3.1) into (1.3) and factoring the denominator, we have [1]

$$
\alpha(\omega)=\frac{\omega+\mathrm{i} \Omega}{m\left(\omega+\mathrm{i} \Omega^{\prime}\right)\left(\omega_{0}^{2}-\omega^{2}-\mathrm{i} \gamma \omega\right)},
$$

where the introduced parameters $\Omega^{\prime}, \omega_{0}$ and $\gamma$ satisfy the relations

$$
\frac{1}{\Omega}=\frac{1}{\Omega^{\prime}}+\frac{\gamma}{\omega_{0}^{2}}, \quad \frac{K}{M}=\frac{\omega_{0}^{2} \Omega^{\prime}}{\Omega^{\prime}+\gamma}, \quad \frac{M}{m}=\frac{\left(\omega_{0}^{2}+\gamma \Omega^{\prime}\right)\left(\Omega^{\prime}+\gamma\right)}{\omega_{0}^{2} \Omega^{\prime}},
$$

where

$$
M=m+2 e^{2} \Omega / 3 c^{3}
$$

is the renormalized mass. 
In partial fraction form, (3.2) becomes

$$
\alpha(\omega)=\frac{A}{\omega+\mathrm{i} \Omega^{\prime}}+\frac{B}{\omega+\mathrm{i} \omega_{1}}+\frac{C}{\omega+\mathrm{i} \omega_{2}},
$$

where

$$
\omega_{1,2}=\frac{1}{2} \gamma \pm \mathrm{i} \sqrt{\omega_{0}^{2}-\frac{1}{4} \gamma^{2}}
$$

and

$$
\begin{aligned}
& A=\frac{\mathrm{i}\left(\Omega-\Omega^{\prime}\right)}{m\left(\omega_{0}^{2}+\Omega^{\prime 2}-\gamma \Omega^{\prime}\right)}, \quad B=\frac{\mathrm{i}\left(\Omega-\omega_{1}\right)}{m\left(\Omega^{\prime}-\omega_{1}\right)\left(\omega_{2}-\omega_{1}\right)}, \\
& C=\frac{\mathrm{i}\left(\Omega-\omega_{2}\right)}{m\left(\Omega^{\prime}-\omega_{2}\right)\left(\omega_{1}-\omega_{2}\right)} .
\end{aligned}
$$

From (3.7), it can be readily shown that

$$
A+B+C=0 .
$$

The imaginary part of $\alpha(\omega)$, from (3.5), is

$$
\operatorname{Im} \alpha(\omega)=\omega \operatorname{Im}\left(\frac{A}{\omega^{2}+\Omega^{\prime 2}}+\frac{B}{\omega^{2}+\omega_{1}^{2}}+\frac{C}{\omega^{2}+\omega_{2}^{2}}\right) .
$$

In the large-cutoff limit $\left(\Omega^{\prime} \gg \gamma\right.$ and $\left.\Omega^{\prime} \gg \omega_{0}\right)$, the first terms in (3.5) and (3.9) are negligible and we remark that similar limits are obtained if one first took the large-cutoff limit in $\alpha(\omega)$ itself.

Substituting (3.9) into (1.6) and using (3.8), we obtain

$$
\left.\left\langle x^{2}\right\rangle\right|_{T-0}=\frac{\hbar}{\pi} \int_{0}^{\infty} \mathrm{d} \omega \operatorname{Im} \alpha(\omega)=-\frac{\hbar}{\pi} \operatorname{Im}\left(A \ln \Omega^{\prime}+B \ln \omega_{1}+C \ln \omega_{2}\right) .
$$

Now we may pass to the large-cutoff limit $(m \rightarrow 0)$, which from (3.3), (3.4) and (3.7) can be shown to give

$$
\Omega^{\prime}=\frac{M}{m \tau_{e}}\left[1+\mathrm{o}\left(\frac{m}{M}\right)\right], \quad \omega_{0}^{2}=\frac{K}{M}+\mathrm{o}\left(\frac{m}{M}\right), \quad \gamma=\omega_{0}^{2} \tau_{e}+o\left(\frac{m}{M}\right),
$$

where $\tau_{e} \equiv 2 e^{2} / 3 M c^{3} ;$ and 


$$
\begin{aligned}
& A=-\frac{\mathrm{i} \tau_{e}}{M}+\mathrm{o}\left(\frac{m}{M}\right), \quad B=\frac{\mathrm{i} \omega_{1}^{2}}{M \omega_{0}^{2}\left(\omega_{1}-\omega_{2}\right)}+\mathrm{o}\left(\frac{m}{M}\right), \\
& C=\frac{\mathrm{i} \omega_{2}^{2}}{M \omega_{0}^{2}\left(\omega_{2}-\omega_{1}\right)}+\mathrm{o}\left(\frac{m}{M}\right) .
\end{aligned}
$$

The omitted terms are all of the order of $m / M$. From the last of equations (3.11), it is clear that $\gamma$ is a function of $\omega_{0}$, and hence the only independent parameters in this problem are $T$ and $\omega_{0}$. In fact for $m \rightarrow 0$, it is clear from (3.10) to (3.12) that the integral expression for $\left\langle x^{2}\right\rangle$ in the blackbody radiation case is the same as that in the Ohmic case except for an extra factor of $\omega^{2} / \omega_{0}^{2}$ in the integrand, which results in a linear divergent integral in the blackbody case. However, if the integration is performed before the large-cutoff limit is taken (which is the preferred procedure), then a logarithmically divergent result is obtained (see (3.13)).

Let us, first of all, examine the zero temperature case. Using (3.11) and (3.12) in (3.10), we obtain

$$
\begin{aligned}
\left.\left\langle x^{2}\right\rangle\right|_{T=0}= & \frac{\hbar \tau_{e}}{\pi M} \ln \left(\frac{M}{m}\right)-\frac{\hbar \tau_{e}}{\pi M} \ln \tau_{e} \\
& +\frac{\hbar}{2 \pi M \omega_{0}^{2}} \operatorname{Im}\left(\frac{1}{\sqrt{\omega_{0}^{2}-\frac{1}{4} \gamma^{2}}}\left[\omega_{2}^{2} \ln \omega_{2}-\omega_{1}^{2} \ln \omega_{1}\right]\right) \\
= & \frac{\hbar \tau_{e}}{\pi M} \ln \left(\frac{M}{m}\right)-\frac{\hbar \tau_{e}}{\pi M} \ln \left(\omega_{0} \tau_{e}\right) \\
& +\frac{\hbar}{\pi M \omega_{0}^{2}}\left[\frac{\omega_{0}^{2}-\frac{1}{2} \gamma^{2}}{\sqrt{\omega_{0}^{2}-\frac{1}{4} \gamma^{2}}} \tan ^{-1}\left(\frac{2}{\gamma} \sqrt{\omega_{0}^{2}-\frac{1}{4} \gamma^{2}}\right)\right]
\end{aligned}
$$

The first term in the above equation, though logarithmically divergent as $m \rightarrow 0$, is independent of $K$ (or $\left.\omega_{0}^{2}\right)$, and therefore $\left.(\partial / \partial K)\left\langle x^{2}\right\rangle\right|_{T=0}$ is finite:

$$
\begin{aligned}
\left.\frac{\partial}{\partial K}\left\langle x^{2}\right\rangle\right|_{T=0}= & -\frac{\hbar}{2 \pi K^{2}}\left[\frac{\omega_{0}^{2}}{\left(\omega_{0}^{2}-\frac{1}{4} \gamma^{2}\right)^{3 / 2}} \tan ^{-1}\left(\frac{2}{\gamma} \sqrt{\omega_{0}^{2}-\frac{1}{4} \gamma^{2}}\right)\right. \\
& \left.+\frac{\gamma\left(3 \omega_{0}^{2}-\gamma^{2}\right)}{2\left(\omega_{0}^{2}-\frac{1}{4} \gamma^{2}\right)}\right]
\end{aligned}
$$

which is negative by the inequalities

$$
\tan ^{-1} x>x\left(1-3 x^{2}\right) /\left(1+x^{2}\right)^{2} \quad(x>0)
$$

and 


$$
\frac{1}{2} \ln [(1+x) /(1-x)]<x\left(1+3 x^{2}\right) /\left(1-x^{2}\right)^{2} \quad(0<x<1) .
$$

In other words, in the case of a blackbody radiation heat bath at zero temperature, localization is enhanced due to increased $K$.

Expressions (3.13) and (3.14) are written in the form valid for $\omega_{0}>\frac{1}{2} \gamma$. In the case of $\omega_{0}<\frac{1}{2} \gamma$, one needs just to replace $\left(\omega_{0}^{2}-\frac{1}{4} \gamma^{2}\right)^{-1 / 2} \tan ^{-1}[(2 /$ $\left.\gamma)\left(\omega_{0}^{2}-\frac{1}{4} \gamma^{2}\right)^{1 / 2}\right]$ by

$$
\frac{1}{\sqrt{\frac{1}{4} \gamma^{2}-\omega_{0}^{2}}} \frac{1}{2} \ln \left(\frac{\frac{1}{2} \gamma+\sqrt{\frac{1}{4} \gamma^{2}-\omega_{0}^{2}}}{\frac{1}{2} \gamma-\sqrt{\frac{1}{4} \gamma^{2}-\omega_{0}^{2}}}\right),
$$

according to the identity

$$
\tan ^{-1}(\mathrm{i} x)=\frac{1}{2} \mathrm{i} \ln [(1+x) /(1-x)] .
$$

We now turn to the case of non-zero temperature. For the temperature dependent part of $\left\langle x^{2}\right\rangle$, the contribution due to the first term of $\operatorname{Im} \alpha(\omega)$ in (3.9), when inserted in (2.7), is

$$
\frac{\hbar}{\pi} \operatorname{Im}\left\{A\left[\ln \left(\frac{\hbar \Omega^{\prime}}{2 \pi k T}\right)-\psi\left(\frac{\hbar \Omega^{\prime}}{2 \pi k T}\right)-\frac{\pi k T}{\hbar \Omega^{\prime}}\right]\right\},
$$

which, by using the asymptotic expansion of $\psi(z)$,

$$
\psi(z) \sim \ln z-\frac{1}{2 z}-\frac{1}{12 z^{2}} \cdots \quad(z \rightarrow \infty \text { in }|\arg z|<\pi),
$$

approaches to

$$
\frac{\hbar}{\pi} \operatorname{Im}\left[\frac{A}{12}\left(\frac{2 \pi k T}{\hbar \Omega^{\prime}}\right)^{2}\right] \rightarrow 0 \quad \text { as } \Omega^{\prime} \rightarrow \infty \quad(\text { or } m \rightarrow 0) .
$$

Thus, to calculate $\Delta\left\langle x^{2}\right\rangle$, one might simply take the large-cutoff limit in $\alpha(\omega)$ first before the integration. This is valid here because the resulting integral is finite, hence the order of limiting and integrating can be exchanged.

Combining the remaining two terms in (3.9) with (2.8) and (2.11), we obtain

$$
\begin{aligned}
\Delta\left\langle x^{2}\right\rangle= & \frac{2 \pi(k T)^{2}}{\hbar M \omega_{0}^{2}} \operatorname{Im}\left(\frac { 1 } { \sqrt { \omega _ { 0 } ^ { 2 } - \frac { 1 } { 4 } \gamma ^ { 2 } } } \left[z_{1}^{2} \ln z_{1}-z_{1}^{2} \psi\left(z_{1}\right)-\frac{1}{2} z_{1}\right.\right. \\
& \left.\left.-z_{2}^{2} \ln z_{2}+z_{2}^{2} \psi\left(z_{2}\right)+\frac{1}{2} z_{2}\right]\right),
\end{aligned}
$$


where $z_{1}$ and $z_{2}$ are again given by (2.10), with $\gamma$ understood to be satisfying (3.11).

In the high-temperature limit, this expression becomes

$$
\Delta\left\langle x^{2}\right\rangle=\frac{k T}{M \omega_{0}^{2}}-\frac{\hbar \tau_{e}}{\pi M} \ln \left(\frac{2 \pi k T}{\hbar \omega_{0}}\right)+\cdots,
$$

while in the low-temperature limit it becomes

$$
\Delta\left\langle x^{2}\right\rangle=\frac{2 \pi^{3} \tau_{e}(k T)^{4}}{15 M \hbar^{3} \omega_{0}^{4}}+\cdots
$$

Next consider the $\omega_{0} \tau_{e} \ll 1$ limit, which is true in most circumstances, since $\tau_{e}$ is typically exceedingly small $\left(\tau_{e}=2 \times 10^{-24} \mathrm{~s}\right.$ in the case of the electron). Then

$$
\left.\left\langle x^{2}\right\rangle\right|_{T=0}=\frac{\hbar \tau_{e}}{\pi M} \ln \left(\frac{M}{m \omega_{0} \tau_{e}}\right)+\frac{\hbar}{\pi M \omega_{0}}\left[\frac{1}{2} \pi-\frac{1}{2} \omega_{0} \tau_{e}-\frac{3}{16} \pi\left(\omega_{0} \tau_{e}\right)^{2}+\cdots\right]
$$

and

$$
\begin{aligned}
\Delta\left\langle x^{2}\right\rangle= & \frac{\hbar}{M \omega_{0}\left[\exp \left(\hbar \omega_{0} / k T\right)-1\right]}+\left(\omega_{0} \tau_{e}\right)\left[\frac{\hbar}{\pi M \omega_{0}} \ln \left(\frac{\hbar \omega_{0}}{2 \pi k T}\right)\right. \\
& \left.+\frac{\hbar}{2 \pi M \omega_{0}}-\frac{\hbar}{\pi M \omega_{0}} \operatorname{Re}\left[\psi\left(z_{0}\right)+\frac{1}{2} z_{0} \psi^{\prime}\left(z_{0}\right)\right]\right]+o\left(\omega_{0} \tau_{e}\right)^{2},
\end{aligned}
$$

where $z_{0} \equiv \mathrm{i} \hbar \omega_{0} / 2 \pi k T$.

Finally, taking the derivative of $\left\langle x^{2}\right\rangle$ with respect to $K$, we have

$$
\begin{aligned}
\frac{\partial}{\partial K}\left\langle x^{2}\right\rangle= & \left.\frac{\partial}{\partial K}\left\langle x^{2}\right\rangle\right|_{T=0}+\frac{\partial}{\partial K} \Delta\left\langle x^{2}\right\rangle \\
= & \frac{\hbar}{4 \pi K^{2}\left(\omega_{0}^{2}-\frac{1}{4} \gamma^{2}\right)} \operatorname{Im}\left(\mathrm{i}\left[z_{2} \omega_{2}^{3} \psi^{\prime}\left(z_{2}\right)+z_{1} \omega_{1}^{3} \psi^{\prime}\left(z_{1}\right)\right]\right. \\
& \left.+\frac{\omega_{0}^{4}}{\sqrt{\omega_{0}^{2}-\frac{1}{4} \gamma^{2}}}\left[\psi\left(z_{2}\right)-\psi\left(z_{1}\right)\right]\right)+\frac{k T}{K^{2}}
\end{aligned}
$$

By the same technique used in section 2 , one may show that

$$
\frac{\partial}{\partial K}\left\langle x^{2}\right\rangle=-\frac{2 k T}{K^{2}}\left[\frac{1}{2}+\sum_{n=1}^{\infty}\left(\frac{\left(z_{1}+z_{2}\right) n+z_{1} z_{2}}{\left(n+z_{1}\right)\left(n+z_{2}\right)}\right)^{2}\right]<0 .
$$

We conclude that, in the case of a blackbody radiation heat bath, at any temperature, the localization is enhanced due to increasing $K$ (i.e. increased 
binding). A similar result holds in the case of increasing dissipation since $\gamma$ is proportional to $K$.

\section{Conclusions}

In the case of an arbitrary spectral distribution we have shown that localization increases with decreasing temperature. Also, we have shown that, in the case of an Ohmic heat bath and a blackbody radiation heat bath, at any temperature, either increase of dissipation or increase of binding leads to an enhancement of localization.

\section{Acknowledgements}

This research was partially supported by the U.S. Office of Naval Research under Contract no. N00014-90-J-1124 and by the National Science Foundation Grant no. INT-890-2519. One of us (RFO'C) would like to thank Dr. Peter Knight for encouraging him to do this problem some years ago.

\section{References}

[1] G.W. Ford, J.T. Lewis and R.F. O'Connell, Phys. Rev. Lett. 55 (1985) 2273; Phys. Rev. A 36 (1987) 1466.

[2] G.W. Ford, J.T. Lewis and R.F. O'Connell, Phys. Rev. A 37 (1988) 4419.

[3] A.O. Caldeira and A.J. Leggett, Ann. Phys. (NY) 149 (1983) 374.

[4] G.W. Ford, J.T. Lewis and R.F. O'Connell, Ann. Phys. (N.Y.) 185 (1988) 270.

[5] M. Abramowitz and I.A. Stegun, eds., Handbook of Mathematical Functions (Dover, New York, 1964) p. 259. 